\title{
La gobernanza y la gestión de las instituciones culturales nacionales:* de la oposición entre arte y economía a la articulación entre política cultural y gestión
}

\author{
Joaquim Rius Ulldemolins \\ Universitat de Barcelona. Departament de Teoria Sociològica, Filosofia del Dret \\ i Metodologia de les Ciències Socials \\ joaquim.rius@ub.edu
}

\section{Resumen}

En el siglo XX, el debate en torno a la gobernanza y la gestión de las instituciones culturales nacionales ha sido centrado en buena medida en la relación problemática entre el arte y la economía. No obstante, diversos cambios han hecho que éste sea un debate desfasado. La pérdida de autonomía del mundo artístico, la transformación de la producción y de la difusión cultural o la instrumentalización de las políticas culturales generan un nuevo contexto en el que irrumpe la figura del gerente artístico. Actualmente, la gobernanza y la gestión de las instituciones culturales nacionales se sitúa en una articulación problemática entre la política cultural y el agente de arte que sustituye al binomio anterior formado por arte versus economía. El artículo mostrará este cambio en el caso de Barcelona, analizando los discursos de los responsables políticos y los de los directores de las instituciones culturales nacionales.

Palabras clave: instituciones culturales nacionales; dirección de arte; política cultural; instrumentalización.

* Con la denominación «instituciones culturales nacionales», englobamos aquellas organizaciones dirigidas a la producción y exhibición de cultura especializada de todos los sectores artísticos que se orientan a una proyección extralocal o regional, superan el millón de euros de presupuesto y cuentan con una participación de las administraciones públicas en sus órganos de gobierno y en la financiación de su actividad regular. En este artículo, no queremos entrar en la utilización política del término nacional en el contexto de Cataluña, de modo que usamos el término nacional en su acepción anglosajona, para distinguirla de las instituciones culturales del sector privado lucrativo o del tercer sector, sin por ello entrar en la denominación de institución cultural pública, al ser problemático usarla en el sector cultural (Schuster, 1998).

El autor quiere agradecer los consejos del profesor Vincent Dubois (Université de Strasbourg) sobre el contenido y orientación del artículo. Asimismo, quiere agradecer los comentarios de los revisores de la revista. Finalmente, damos también las gracias a los responsables de las políticas culturales y de las instituciones culturales por dedicarnos su tiempo en las entrevistas que fundamentan el artículo. 
Abstract. The governance and management of national cultural institutions: From the opposition between art and economy to the relationship between cultural policy and management

In the twentieth century the debate on the governance and management of national cultural institutions has largely focused on the problematic relationship between art and the economy. Several changes, however, have made this an outdated discussion. The loss of autonomy of the art world, the transformation of cultural production and distribution, and the instrumentalization of cultural policies have given rise to a new context in which art managers play a key role. Currently, the governance and management of national cultural institutions is at a problematic crossroads between cultural policy and art management, thus replacing the previous coupling of art versus economy. The article shows these changes in the case of Barcelona and analyzes the discourses of politicians and managers of national cultural institutions.

Keywords: national cultural institutions; art management; cultural policy; instrumentalization

\begin{aligned} & \multicolumn{2}{c}{ Sumario } \\ & 1. Introducción 3. Conclusiones \\ & $\begin{aligned} & \text { 2. La articulación entre responsables } \text { Referencias bibliográficas } \\ & \text { políticos y mánagers de las instituciones } \\ & \text { culturales nacionales: } \\ & \text { un análisis cualitativo }\end{aligned}\end{aligned}$

\section{Introducción}

\subsection{Las instituciones culturales como objeto de estudio}

El análisis sobre la dirección de las instituciones culturales nacionales ha ido ganando peso en el mundo académico, a medida que crecía su número y su dimensión y se convertían en una de las principales herramientas de la política cultural (Coppinger et al., 2007) y en un instrumento para la consecución de objetivos de otras áreas de gobierno, como la regeneración urbana, la promoción económica o el desarrollo de la cohesión social (Bianchini, 1993; Gray, 2007).

El estudio de la gestión de las instituciones culturales se ha focalizado, habitualmente, en la figura del director de arte o, en su denominación anglosajona, el art manager, caracterizado como una figura opuesta a la del artista. Tradicionalmente, se ha conceptualizado el director de arte como el profesional que garantiza la viabilidad económica de un proyecto artístico. Diversos autores han destacado su influencia en la actividad artística (Kuesters, 2010) y han caracterizado el rol de dicho gestor cultural como una figura doble (Cray et al., 2007), que fusiona las lógicas del mundo artístico y la esfera económica (Chiapello, 1998). Sin embargo, el rol de los directores de arte se ha transformado en gran medida: si en los años ochenta podíamos hablar de revolución en la gestión de las instituciones culturales (DiMaggio, 1991; Peterson, 1986), actualmente, desde nuestro punto de vista, podemos hablar de un giro en la gobernanza de las instituciones culturales. 
Este artículo parte de la hipótesis que, para analizar correctamente la gobernanza y la gestión de las instituciones culturales nacionales, debemos considerar la lógica de esferas sociales distintas: la artística, la económica y también la político-institucional. En este sentido, retomamos la idea de Max Weber sobre la separación de esferas sociales que caracteriza a la modernidad (Weber, 1944; Weber, 1998) y que orienta el mundo artístico hacia una dinámica crecientemente autónoma y autorreferencial (Becker, 1984). Una dinámica que se iniciará a mediados del siglo XX, con la crisis del sistema académico y el establecimiento del mercado del arte (White y White, 1991), y que perdurará hasta finales del siglo XX, con la irrupción de las dinámicas postmodernas, en que se desarrolla un proceso de desdiferenciación de las esferas sociales (Lash, 1990).

Asimismo, creemos, siguiendo a Rodríguez Morató, que esta menor separación entre las esferas sociales comporta que, actualmente, el sector artístico sea menos autónomo y, por lo tanto, reciba muchas más influencias y esté más condicionado por el campo económico y político (Rodríguez Morató, 2007). Por otra parte, entendemos, como Crozier y Friedberg (1982), que la acción del actor social no está determinada por el sistema, sino que existe un margen de maniobra en el que se desarrollan estrategias para conseguir objetivos dentro de un marco dinámico. Por lo tanto, debe entenderse que las acciones y los discursos de los responsables políticos y los mánagers artísticos no están determinados por el contexto, sino que constituyen juegos estratégicos para definir posiciones de poder, influencia y prestigio dentro del mundo cultural.

En todo caso, mostraremos como hay una tendencia en diversos países en la que los responsables políticos están protagonizando un rol más preponderante en la definición de la política cultural (Dubois et al., 2012; Gray, 2008) y en la que el mánager tiene un papel más activo en la gestión de los equipamientos culturales nacionales (Cray et al., 2007; Dewey, 2004; Peterson, 1986). Esta tendencia se expresa en el caso de Barcelona, que es un caso singular y avanzado de la política cultural (Rius, 2005; Rodriguez Morató, 2008).

Para elaborar este artículo, nos hemos basado en las entrevistas realizadas entre 2008 y 2012, en el marco de la investigación titulada El sistema de la política cultural en España ${ }^{1}$. Por una parte, se ha entrevistado a los máximos responsables de las principales instituciones culturales de Barcelona: el Museu Nacional d'Art de Catalunya, el Museu d'Art Contemporani de Barcelona, el Teatre Nacional de Catalunya, el Teatre Lliure, el centro de danza Mercat de les Flors, el Auditori de Barcelona, el Palau de la Música, la Fundació Joan Miró y la Fundació Antoni Tàpies².

1. Proyecto financiado por el Plan Nacional de Investigación del Ministerio de Educación (Ref. CSO2008-05910/SOCI).

2. Se ha realizado una entrevista semidirigida a los directores de las principales instituciones culturales barcelonesas, excepto el Gran Teatre del Liceu. Su director declinó ser entrevistado, a causa de la grave crisis económica e institucional que padeció en la temporada 2011-2012, y se entrevistó a los representantes de la plataforma Salvem el Liceu, que integra a miembros de la sociedad civil y a los empleados del teatro lírico. El resultado de las entrevistas ha sido transcrito y su contenido ha sido analizado de forma específica para el presente artículo. 
Por otra parte, se ha entrevistado también a seis responsables políticos de la gestión cultural del Gobierno autonómico catalán y del Ayuntamiento de la ciudad de Barcelona. Se ha elegido a antiguos y actuales responsables para representar una diversidad de experiencias y colores políticos. En total, se ha entrevistado a dieciséis personas, un universo que se considera representativo de la diversidad de posiciones políticas y sectoriales sobre la gobernanza y la gestión de las grandes instituciones culturales ${ }^{3}$.

En primer lugar, analizaremos la influencia de los cambios sociales y económicos que, desde finales del siglo XX, han transformado la política cultural, la gobernanza y la gestión de las instituciones culturales nacionales, con el surgimiento de la dirección de arte y la instrumentalización de las políticas culturales. En segundo lugar, presentaremos brevemente el desarrollo de las instituciones culturales nacionales en Barcelona. Y, en tercer lugar, analizaremos los cambios en la gobernanza y la gestión de estas instituciones a partir de las entrevistas cualitativas a sus responsables políticos y sus directivos. Ello nos permitirá analizar los puntos de consenso y los conflictos sobre la capacidad de gobernar a las instituciones culturales y sobre la legitimidad de definir sus objetivos y sus planes de gestión. Finalmente, concluiremos con unas reflexiones finales acerca de la centralidad del gestor cultural en el nuevo equilibrio entre la esfera artística, la económica y la política, en el marco del debate en el campo de la sociología de la cultura y el estudio de las políticas culturales.

\subsection{Cambios socioeconómicos, política cultural y gobernanza de las instituciones culturales}

La investigación sobre la gestión de las grandes organizaciones artísticas ha avanzado mucho en los últimos treinta años (Evrard y Colbert, 2000). Uno de los avances principales en el análisis de la gobernanza de las grandes instituciones culturales es destacar la influencia del marco social y político en los procesos de privatización (Mulcahy, 1998; Mulcahy, 2003), en la mezcla de titularidades públicas y privadas (Rius, 2012b; Schuster, 1998) o en su orientación hacia objetivos sociales (Belfiore, 2004).

No obstante, uno de los aspectos que condicionan la gobernanza y la gestión de las instituciones culturales nacionales es el grado de intervención de las administraciones públicas. Como señalan Zimmer y Toepler (1999), el

3. Ciertamente, el rol del gerente cultural puede ser percibido de una forma diferente en función de la posición que se ocupe dentro del mundo artístico. La ambivalente relación de los marchantes de arte, los artistas y las instituciones culturales ha sido analizada por la socióloga del arte Raymonde Moulin en su estudio sobre el mercado del arte y la política cultural en Francia (Moulin, 1992). Esta autora sostiene que existe una mayor relación e interdependencia simbólica y económica entre el mercado y la institución, que se refleja en una proximidad espacial entre los distritos de galerías y los museos de arte (íbídem). Una alianza que ha sido verificada también en Barcelona y que tiene efectos palpables en los procesos de regeneración urbana (Rius, 2006; Rius, 2012a). Sin embargo, el presente artículo se centrará en analizar las relaciones entre los directivos de las instituciones culturales y los responsables políticos, como una relación central en la definición de la misión de la organización. 
grado de implicación del estado en la política cultural, en general, y de las grandes instituciones culturales, en particular, se explican por las configuraciones político-institucionales que se han desarrollado de forma distinta en los países desarrollados. En el mundo anglosajón, la tradición de intervención del estado en el mundo cultural es más débil y se vehicula mediante organismos independientes (el principio del arm's length). En este caso, las instituciones culturales son jurídicamente independientes del sector público y existe una fuerte tradición de participación de mecenazgo. Por el contrario, en la Europa continental, la configuración institucional desde la posguerra establece que el estado tenga un rol preponderante en la gestión de la política cultural. En este caso, las instituciones culturales nacionales son altamente dependientes del sector público, de modo que el estado es su principal y a veces único financiador.

Consecuencia de este modelo de política cultural europeo-continental se ha producido, durante la segunda mitad del siglo XX, una situación paradójica en las instituciones culturales nacionales: una gran dependencia económica de las administraciones públicas combinada con un alto grado de autonomía en la formulación de la actividad. Una configuración legitimada por el discurso generado en el mundo artístico (Becker, 1984; Moulin, 1983) y por la tradición que se establece a nivel internacional a mediados del siglo XIX de unas instituciones culturales de élite orientadas a la excelencia artística (Martorella, 1982). Tal situación ha perdurado hasta finales del siglo XX.

\subsection{El nacimiento de las grandes instituciones culturales y la oposición entre dirección artística y gestión}

Las grandes instituciones culturales son producto de su etapa histórica y están ligadas a la élite económica y social que las impulsa. A su vez, a cada etapa histórica, le ha correspondido una forma de dirección (Agid y Tarondeau, 2007). En el Antiguo Régimen, el director era un sirviente más de la corte obligado a satisfacer sus gustos y, durante el siglo XIX, éste se convierte en un empleado dependiente de los patronos privados o de los mecenas institucionales (Agid y Tarondeau, 2006). No será hasta fines del siglo XIX que la mayor pluralidad en la composición de la élite y una ampliación del mercado artístico dará un cierto margen de maniobra a los mánagers y los convertirá en una figura relevante (DiMaggio, 1987a).

A partir de la segunda mitad del siglo XIX, se producirá un proceso de autonomización del mundo del arte que opondrá la dirección artística y la gestión de la institución. La disputa entre el artista y el mánager es una herencia de la oposición cultural entre una idea romántica del arte y la gestión capitalista, considerada entonces como opuesta a lo creativo (Chiapello, 1998). En este contexto, la dirección de las instituciones culturales aparece dividida de forma bipolar: un director artístico consagrado a la misión de la institución cultural orientada a la alta cultura y, en un segundo plano y subordinado, el gerente de la institución dedicado a proporcionar los medios materiales, técnicos y humanos para que tal misión artística pueda realizarse. 
Esta oposición entre lógica artística y lógica empresarial se expresa en una dinámica de enfrentamiento. Por una parte, la dirección artística tiende a fomentar la sobrecalidad de las obras, a fin de ganar reputación profesional y «llenar la sala». Ello deriva en la aparición de déficits crónicos que el gerente debe intentar controlar o, en el caso de no poderlo hacer, recurrir a las administraciones públicas para evitar la bancarrota de la organización, sin poder ofrecer alternativas de gestión (Urfalino, 1989).

Este predominio de los valores artísticos y de la figura del director artístico será común hasta mediados del siglo XX, en el que empiezan a producirse cambios macrosociales que tienen un impacto decisivo en la función social de las grandes instituciones culturales y de su gestión.

\subsection{Cambios macrosociales y transformación del rol de las organizaciones culturales de la alta cultura}

Los cambios en las grandes instituciones culturales están íntimamente ligados a las transformaciones experimentadas por la estructura social y en la relación entre la esfera cultural y la economía. Según DiMaggio (1991), en la segunda mitad del siglo XX, asistimos a tres grandes cambios:

a) El declive de la burguesía comercial e industrial y la irrupción de una élite definida por su posición en las grandes empresas y la estructura estatal, así como el vertiginoso crecimiento de una clase media educada, para la que la cultura es una fuente esencial en la construcción de su identidad y su estatus.

b) El eclipse del patronazgo privado tradicional y el crecimiento de la inversión institucional, sea por parte de las grandes empresas o del estado.

c) Un giro en la gestión de las artes, particularmente en las grandes instituciones de la alta cultura, con la potenciación de la figura del mánager (Peterson, 1986).

Esta transformación se enmarca en un rápido incremento de la financiación pública observable en todos los países avanzados a partir de los años cincuenta, que no se explica por una cuestión de costos (la conocida como la ley de Baumol), sino por el aumento de la dimensión y la cantidad de organizaciones artísticas (DiMaggio, 1987b). El financiamiento institucional erosiona el poder de los patronos privados tradicionales y del sector artístico, expande la definición del público y, paradójicamente, impulsa a las grandes organizaciones artísticas hacia su dependencia del mercado.

En este contexto, podemos identificar tres lógicas de cambio de la gestión de las grandes instituciones culturales respecto a la etapa precedente:

a) Las administraciones públicas y los mecenas institucionales (fundaciones y grandes corporaciones) animan a las organizaciones que reciben sus fondos a incrementar la cantidad de público o, en todo caso, toman el crecimiento 
del público como un indicador de éxito. Esta presión impulsa a las organizaciones artísticas hacia el mercado y hacia el márqueting.

b) Una segunda lógica es la tendencia hacia la rendición de cuentas o la obligación de medir los resultados en el marco de las reformas de la nueva gestión pública (Belfiore, 2004). Ésta es definida en la práctica como la capacidad de generar proyectos de financiación e informes con datos económicos y de público detallados, así como en la creación de estructuras administrativas capaces de satisfacer esta demanda de mayor planificación estratégica. Un efecto de esta lógica es hacer inviables las organizaciones no gestionadas burocráticamente, como los antiguos clubes de la alta cultura. Otro efecto es crear una forma de pericia, lo que representa una fuente de poder en sus conflictos frente a los patronos o el personal artístico. Un tercer efecto es que el incremento de la financiación pública va paralelo al incremento del presupuesto y del personal dedicado a tareas administrativas (DiMaggio, 1987a).

Por todo ello, podemos hablar de un giro en la gestión de las artes, en el sentido de haberse creado una alianza entre los mánagers y los patronos institucionales que altera los modos de funcionamiento y los objetivos de las organizaciones artísticas. La educación y los intereses de los nuevos mánagers los hacen más receptivos a las llamadas al crecimiento, a la reforma de gestión y hostiles al elitismo social o estético.

\subsection{El rol directivo como una tarea creativa orientada a la creación de valor público}

La erosión de la distancia entre las artes y la economía conlleva también un proceso de revalorización de los sectores considerados menores o paraartísticos (diseño, arquitectura), considerados artes menores (fotografía, cómic) o de las profesiones técnicas dentro de los mundos artísticos (Rodríguez Morató, 2007). Una de las profesiones reevaluadas es el rol de mánager, que, hasta mediados del siglo XX, era considerada una profesión técnica subordinada a la voluntad de los mecenas o de los artistas.

A partir de los años ochenta, y especialmente a principios del siglo XXI, se produce un cambio. Los dirigentes de las agencias públicas dejan de ser considerados como simples administradores de un servicio público y pasan a ser conceptualizados como mánagers, que, a partir de una autonomía de gestión, pueden contribuir a la consecución del valor público (Longo, 2007).

En esta redefinición de su rol, se establece que el objetivo del mánager ya no es la simple gestión de un servicio público, sino la creación de valor público para la ciudadanía. El concepto de valor público fue creado por Mark $\mathrm{H}$. Moore (1995) y aplicado por éste a las agencias artísticas públicas (Moore y Moore, 2005) y, aunque es un concepto en debate sobre su aplicabilidad en el campo cultural (Lee et al., 2011), puede ser considerado el valor que los ciudadanos y las ciudadanas otorgan a una organización artística o a su actividad. 
Esta nueva conceptualización atribuye al mánager un rol mucho más activo, en el sentido que, para conseguir esta creación de valor público, es necesario tomar la iniciativa y crear nuevos servicios y líneas de actividad que no habían sido previstas inicialmente en la definición del servicio público. Éste sería el caso de los servicios educativos en las instituciones culturales, una actividad que no formaba parte de la misión fundacional, pero que ha sido desarrollada por parte de los mánagers y que se ha convertido en uno de los principales generadores de valor público de estas instituciones.

Según esta visión, el mánager ya no sería, por lo tanto, una figura contrapuesta a la del artista, ni tampoco un sujeto pasivo de las instrucciones emanadas por parte de los responsables políticos, sino que tendría un rol activo. Es más, partiendo de la definiciones más recientes aplicadas a la creatividad como una capacidad psicológica, algunos autores llegan a discutir en qué medida puede ser considerada creativa una profesión (Bilton y Leary, 2002; Bilton, 2006), lo que les sitúa en una posición central en la gobernanza y la gestión de las instituciones culturales. Según Mulcahy (2003): «one of the key elements in a new wave of cultural entrepreneurship is to move away from a defensive/ reactive leadership style to one that is confident and positive in communicating the incontestable value of the arts and culture for society as a whole».

En este contexto, el nuevo mánager desarrolla las siguientes capacidades de gestión:

a) Reorientar a las instituciones culturales hacia la generación de valor público, creando nuevos servicios y reformulando la misión tradicional ejerciendo un liderazgo estratégico (Cray et al., 2007; Moore, 1995; Moore y Moore, 2005).

b) Adoptar una gestión relacional (Mendoza y Vernis, 2007), referente a las redes culturales internacionales, para fomentar la proyección de la cultura local y el branding territorial (Dewey, 2004; Evans, 2003), el desarrollo de la colaboración y la coproducción con el resto de los agentes del sector cultural (Arts Council England, 2011), así como la interlocución y la atención a las peticiones de las partes interesadas culturales y sociales locales (Stone y Sandfort, 2009).

c) Representar la identidad cultural local en el marco de una creciente diversidad y globalización (Zolberg, 2007).

d) Promover métodos innovadores de desarrollo y fomento de la creatividad (Bilton, 2006), así como de creación de nuevos públicos (Holden, 2008).

e) Potenciar nuevos modelos sostenibles de financiación mixta pública y privada (Asuaga y Rausell, 2006), combinando proyectos de alto valor cultural (y alto riesgo económico), proyectos de alto valor público y proyectos generadores de recursos económicos (Holden, 2004), todo ello en una dinámica de gestión del riesgo artístico y económico similar a la del portafolio inversor (Menger, 2009).

Todas ellas, combinadas en un nuevo rol, constituyen las principales características del giro realizado en la gestión de las instituciones culturales. 


\subsection{Politica cultural, nueva gestión pública e instrumentalización de las grandes organizaciones culturales}

Desde los años ochenta, una de las dos mayores transformaciones de las políticas culturales ha sido su instrumentalización (Gray, 2007). Ésta se puede definir como la utilización de la política cultural para cumplir objetivos de otras áreas de las políticas públicas (Belfiore, 2006). Una de las áreas de la política cultural más afectada por este proceso es la referente a los equipamientos culturales nacionales, unas instituciones que, hasta el momento, consumen una gran parte de los recursos públicos dedicados a la cultura, pero cuya misión, hasta hace veinte años, casi no había evolucionado, puesto que había quedado anclada en la promoción de la excelencia artística (Coppinger et al., 2007).

Una de las primeras instrumentalizaciones de los equipamientos culturales fue su utilización como factor catalizador de los procesos de regeneración urbana a partir de los años ochenta (Bianchini, 1993) y en los procesos de redefinición de la imagen de las ciudades (Evans, 2003). Sin embargo, a partir de los años noventa, y bajo la influencia de la nueva gestión pública (NGP), la política cultural, en general, y los grandes equipamientos culturales, en particular, se orientan hacia el fomento de la cohesión social y la educación artística (Gray, 2008). El giro ha sido parte de la nueva concepción de la política pública orientada a resultados, lo cual, en el caso cultural, ha conllevado dirigir la política cultural a obtener un impacto social (Belfiore, 2004).

Generalmente, esta orientación se ha concebido como una dinámica de arriba a abajo, de los responsables políticos a los equipamientos culturales. Y la forma de implementación ha sido a través de los acuerdos de funcionamiento firmados entre el actor principal (las administraciones públicas o sus arm's length) y el agente, los directivos de las grandes organizaciones culturales. Un proceso que hemos podido observar en distintos países europeos (Rius, 2010).

La aparición de los acuerdos de funcionamiento se sitúa en esta doble voluntad de alinear los objetivos de las agencias públicas con unas políticas públicas orientadas a incrementar el bienestar de los ciudadanos y a legitimarlas mediante la evaluación del retorno social de éstas. Una aparición que se enmarca en los procesos de agencialización impulsados por la NGP y que se están desarrollando en diversos países europeos, entre ellos Cataluña (Rius y Rubio, 2013).

\subsection{Politica cultural e instituciones culturales: el caso de Barcelona}

Tal y como analiza Arturo Rodríguez (2008; 2005), Barcelona ha desarrollado una intensa estrategia cultural para facilitar su desarrollo urbano desde el siglo XIX hasta nuestros días. Esta estrategia se refuerza con la fuerte presencia de la identidad catalana, puesto que la ciudad ejerce de capital de dicha cultura (Villarroya, 2012). Asimismo, a partir de los años ochenta, Barcelona desarrolla uno de los modelos más avanzados de ciudad emprendedora (Marshall, 1996) y el Gobierno de la ciudad ha apostado desde entonces por convertir a los sectores culturales y del conocimiento, además del turismo, en vectores 
Tabla 1. Grandes equipamientos culturales, año de fundación y presupuesto (2010)

\begin{tabular}{lcc}
\hline Nombre de la institución cultural nacional & Año de inauguración & $\begin{array}{c}\text { Presupuesto } \\
\text { (miles de euros) }\end{array}$ \\
\hline Gran Teatre del Liceu & 1982 & 55.692 \\
Museu Nacional d'Art de Catalunya & 1991 & 26.493 \\
Auditori de Barcelona & 1999 & 21.022 \\
Teatre Nacional de Catalunya & 1997 & 16.074 \\
Museu d'Art Contemporani de Barcelona & 1995 & 13.315 \\
Teatre Lliure & 2003 & 8.614 \\
Fundació Joan Miró & 1971 & 7.560 \\
Palau de la Música & 1984 & 7.194 \\
Mercat de les Flors & 2008 & 6.646 \\
Fundació Antoni Tàpies & 1994 & 2.170 \\
\hline
\end{tabular}

Fuente: elaboración propia a partir de los datos del Departamento de Cultura del Gobierno autonómico (Departament de Cultura, 2011) y del Ayuntamiento de Barcelona (Ajuntament de Barcelona, 2011).

* En los casos del Liceu, el Teatre Lliure o el Palau de la Música, se ha tomado en consideración no el año de inauguración -ya que fueron creados por iniciativa privada anteriormente-, sino el año de incorporación al sector público.

para el desarrollo de su economía urbana (Trullén, 2001). En este contexto, las instituciones culturales se convertirán en un importante escaparate de la identidad y de la creatividad local y en una bandera de la modernización de la ciudad (Rius, 2006).

A partir de la recuperación de la democracia, el gobierno autonómico catalán y el Ayuntamiento de Barcelona liderarán los proyectos de construcción de equipamientos culturales, al que se incorporará posteriormente el Ministerio de Cultura. Esta dinámica generará un modelo de gobernanza de las instituciones culturales en el que se generalizará la estructura de consorcio entre las tres administraciones públicas. En estos consorcios, las titularidades y las responsabilidades están muy repartidas, pero con preeminencia del nivel regional y local: el Departamento de Cultura financia el 55\%; el Gobierno local, el 25\%, y el Gobierno central, sólo el 20\% (Ajuntament de Barcelona, 2011; Departament de Cultura, 2011).

Por otra parte, se construyen grandes equipamientos que se convierten en una pieza fundamental de regeneración urbana del centro de la ciudad (Rius Uldemollins, 2008) o se crean nuevas zonas de centralidad urbana, como los clústeres culturales de Montjuïc o Poblenou-22@ (Rius, 2005).

La apuesta cultural de Barcelona se puede ver reflejada en la presencia de diez instituciones culturales nacionales (ver tabla 1) de diversos sectores artísticos (patrimonio, arte contemporáneo, teatro, danza y música), la mayoría de las cuales fueron construidas en los años noventa y con unos presupuestos que suman un total de 164,7 millones de euros.

Las aportaciones a las instituciones culturales nacionales representan un 22\% del presupuesto del Departamento de Cultura del año 2010 y un 31\% del presupuesto que el Ayuntamiento de Barcelona destina a dicha partida. Se trata, 
por lo tanto, de uno de los principales instrumentos de la política cultural y un gasto fijo sobre el que sus responsables deben ejercer un rol de tutela y control.

\section{La articulación entre responsables políticos y mánagers de las instituciones culturales nacionales: un análisis cualitativo}

\subsection{La misión de los grandes equipamientos culturales. ¿Quién decide qué?}

Existe un consenso en articular de una forma más explícita la relación entre los responsables políticos y los directivos de las instituciones culturales. No obstante, este consenso es más aparente que de fondo, ya que existen grandes discrepancias en torno a cómo se formula la misión y a los objetivos de estas instituciones.

Para algunos responsables políticos, se debe recuperar la gobernanza de los equipamientos culturales:

Hasta hace poco, los equipamientos culturales del país iban muy por libre. Esto ha permitido que se aislaran de ciertas politizaciones negativas, pero ha llevado a que los políticos ejerciéramos un control muy superficial, sin entrar en los objetivos de fondo. Y las instituciones culturales deben cambiar, porque la sociedad ha cambiado. Por esto, apostamos por el contrato programa, que es un instrumento de las administraciones públicas para ayudar a realizar el cambio. (Entrevista 2. Responsable político del Gobierno autonómico)

Y no solamente se deben redactar los objetivos, sino que también hay que tutelar para que se comuniquen y se cumplan:

Las administraciones públicas deberían estar presentes en las instituciones culturales para hacer el tutelaje, para la corrección de la gestión y el control del presupuesto, y también para que se cumpla lo que hemos escrito en el contrato programa. Las administraciones públicas deben decírselo al director artístico, al presidente y conseguir que se cumpla. (Entrevista 1. Responsable político del Gobierno autonómico)

En general, los mánagers de las grandes instituciones culturales tienden a reclamar una mayor autonomía, a pesar de que reconocen que la intervención de las administraciones públicas en la definición de los objetivos es escasa:

Hasta ahora no tenemos una definición de la misión o de las líneas maestras de actuación. Esto pasa en Holanda, donde yo he trabajado, pero, de momento, aquí no. Aquí, como máximo, en una conversación informal, los responsables políticos te pueden comunicar oralmente las prioridades más importantes. Pero no hay una concreción escrita. (Entrevista 6. Director de institución cultural nacional)

Cuando se les pregunta por la definición de los objetivos, los mánagers los definen en términos de los que tiene la propia institución y no hacen referencia a los contratos programa si no es como derivación del propio plan estratégico: 
Tenemos prácticamente cerrado el plan estratégico del museo y estamos trabajando ya con los interlocutores del Departamento de Cultura para transformar este plan estratégico en un contrato programa. El año 2012 se redactará este contrato programa que desarrollará los objetivos del plan estratégico y, para ello, recibiremos unos medios económicos. Estos objetivos son ambiciosos, a pesar de lo ridículamente limitados que son los medios económicos. Determinaremos una serie de indicadores de cumplimiento: uno será la cifra de público, otro será la procedencia del público, las publicaciones, la recepción de la página web o la generación de recursos propios. (Entrevista 10. Director de institución cultural nacional)

O, en palabras de otro mánager:

Yo lo que tengo clarísimo es que el museo redacta el plan estratégico y lo redacta sólo, libremente. Desde este plan más general, se pueden derivar unos objetivos más concretos que se pueden incluir en el contrato programa. Y la administración participará y pondrá sus exigencias, porque para esto paga. Pero los objetivos en origen deben ser del museo y tienen que ser culturales. (Entrevista 15. Director de institución cultural nacional)

En este sentido, a pesar de que hay un consenso claro sobre la necesidad de formalizar más los objetivos y de adaptarlos a los de valor público (nuevos públicos, vertiente educativa, etc.), existe una oposición entre la visión de los responsables políticos, que ven estos objetivos como una derivación de los programas de gobierno, y la visión de los gerentes, que los ven como una emanación de los objetivos de la organización elaborados internamente.

\subsection{La visión de los responsables políticos. El respeto a la libertad creativa y el gobierno de las políticas culturales}

A pesar de su voluntad de ejercer mayor control y tutela, actualmente, los responsables políticos son conscientes de la necesidad de que los mánagers disfruten de autonomía como un factor de éxito de los equipamientos, por ello, se rechaza la gestión directa.

Parece que los ministros no se han enterado de que el Museo Reina Sofía va bien o va mal en función del director y de si le inyectan más o menos dinero, pero es lo único que pueden hacer, el ministro de Cultura en eso pinta poco y, por lo tanto, lo que debe hacer es establecer marcos reguladores, no intentar intervenir directamente. (Entrevista 6. Responsable político del Gobierno local)

Desde esta perspectiva, el rol del responsable político es el de crear un marco adecuado sin una dependencia orgánica de la Administración, lo que significa un cambio de perspectiva coherente con la nueva gestión pública. Se considera que una condición para el éxito de la gobernanza de las instituciones culturales nacionales depende de otorgar mayor autonomía jurídica y financiera, así como de fomentar una gestión basada en los resultados. 
Debemos basarnos en este tipo de nuevas políticas en las que la Administración pasa a asumir un papel de catalizador del sector cultural y actúa sobre los efectos, no sobre la producción directa de los servicios. Ahora que estamos en un momento de incertidumbre e inseguridades, lo más fácil es volverse a agarrar a lo tangible y que el ministro o el concejal de Cultura quiera entrar en la gestión de las instituciones para poder decir: «Mire, yo no sé lo que está ocurriendo en el mundo, pero mi museo o mi auditorio funcionan estupendamente gracias a mí». Pero eso no funcionaría, hay que dar autonomía de gestión. (Entrevista 4. Responsable político del Gobierno local)

El esquema actual de gobernanza de los grandes equipamientos ha tendido hacia un esquema estándar en el que existe un consejo de tipo institucional representativo y una comisión de carácter ejecutivo. En realidad, según las opiniones de la mayoría de los entrevistados, estas estructuras refrendan más las decisiones del mánager que decide proactivamente. Éste último, que es nombrado para que ejerza su cargo a lo largo de diversas temporadas (habitualmente tres o cuatro años), dispone de amplios márgenes de maniobra.

El órgano de gobierno es un consejo general presidido por el Honorable Presidente de la Generalitat y por el alcalde de Barcelona que se reúne anualmente. [...] El director es responsable delante de este consejo y tiene un gerente que depende del director. Al director, se le exigen cuentas, no puede tener déficit, no puede perder visitantes, tiene que construir una buena imagen con la prensa. Pero en el consejo no se discute si una exposición se tiene o no que hacer. Estas cosas no se tratan, se confía en el director, que para eso lo han elegido. Es como con los hospitales, se conciben unos servicios y, dentro de cada servicio, se deja hacer al médico. Así es como ha funcionado el sistema, da libertad y, a cambio, se exigen resultados. (Entrevista 9. Director de institución cultural nacional)

\subsection{La emergencia del rol de mánager y la creación de valor público}

La oposición que había tradicionalmente en las instituciones culturales y que estaba representada en las figuras del director artístico y el gerente económico está siendo reemplazada por la de un director plenipotenciario que conjuga la vertiente artística y de gestión. Este es el caso de ocho de las diez instituciones culturales analizadas. En esta ocasión, el director se compara con un profesional con una amplia autonomía basada en un campo de conocimiento especializado, como, por ejemplo, los médicos, y al que los responsables políticos otorgan confianza al nombrarlo.

Una confianza que también se transforma en una mayor autonomía económica, puesto que se pasa del control ex ante a controles ex post más propios de la nueva gestión pública.

Hasta hace dos años, el museo era objeto de una intervención previa para ejecutar los pagos. La Administración pública fiscalizaba cada euro que nos 
daba. A partir de entonces, eso ha cambiado, porque el museo ha madurado como institución y merece la confianza de las administraciones públicas. Desde entonces se realiza una auditoria posterior. (Entrevista 12. Director de institución cultural nacional)

Pero también hay algunos mánagers que reivindican, con límites, la libertad basada en la autonomía del mundo artístico y su capacidad de innovación:

Las instituciones culturales están basadas en la libertad de sus responsables, libertad interpretable, que se ejerce de acuerdo con unas condiciones. No es una carta blanca para hacer lo que te salga de las narices. Si no tienes libertad de pensamiento ni capacidad para reinventar las cosas, no haces tu tarea. No son contables, son poetas. (Entrevista 8. Director de institución cultural nacional)

Una libertad que puede comportar una redefinición entera de las líneas de actividad por parte de la institución cultural y para potenciar su valor público.

Ahora estamos elaborando el plan estratégico. La sensación es la de tener un museo con un potencial enorme no explotable y muchos frentes abiertos. La idea es desplegar todas las funcionalidades que se suponen a un museo e ir más allá. El objetivo para esta etapa es un museo mucho más social, un lugar más público, un lugar de encuentro, de debate. Los museos en general o ganan centralidad social o quedarán apartados. [...] Tengo la impresión de que todos los esfuerzos que se han hecho hasta ahora se habían dirigido a abrir sus puertas, pero no para proyectarse hacia fuera. Por eso hay un gran potencial de crecimiento. (Entrevista 16. Director de institución cultural nacional)

Otro mánager va más allá en la redefinición de la misión de las instituciones culturales afirmando, no sin cierta polémica:

Serán sociales y educativas o no serán. (Entrevista 14. Director de institución cultural nacional)

En esta línea, los mánagers toman la iniciativa por delante de los responsables políticos, con lo cual demuestran una notable capacidad de innovación que pretende hacer crecer sus organizaciones, tal y como subraya DiMaggio (1987a). Una capacidad de innovación que, a veces, llega a intentar redefinir aspectos tan fundamentales de la institución cultural como su nombre mismo.

Todo el mundo conoce la Tate de Londres o el Louvre de Paris, pero con este nombre que tenemos es muy difícil ir por el mundo y hacerse conocer. El nombre del museo no quiere decir nada. Habría que cambiarlo y, seguramente, introducir una referencia a la marca Barcelona, que sí que es conocida internacionalmente. (Entrevista 16. Director de institución cultural nacional) 
Una pretensión que, tras una polémica en la prensa ${ }^{4}$, fue descartada por los responsables políticos, aunque ésta mostró que no está claro quién tiene potestad para decidir aspectos fundamentales de los equipamientos culturales.

\subsection{Procesos de racionalización y legitimidad de actuación en el campo}

Una de las capacidades que reivindican los responsables políticos es establecer, según una racionalidad de la gestión pública, una reordenación de la gestión de los equipamientos culturales. Esta reordenación, que pretende conseguir un ahorro económico y, a la vez, una mayor capacidad de control por parte de los gobiernos, consistiría en la fusión de las instituciones culturales nacionales según criterios propios de la gestión pública, y no de sectores artísticos. A raíz de una propuesta en este sentido, se ha desarrollado un debate sobre la conveniencia y la legitimidad de desarrollar este tipo de proyectos:

Ahora, el consejero, Ferran Mascarell, quiere unificar el MACBA, el CCCB y la Filmoteca, proyecto que me parece negativo, pero aún me parece más negativo que un consejero no lo pueda hacer. Porque, si no puede reordenar el mapa de equipamientos públicos, ¿qué puede hacer? Hoy en día, el consejero necesita la aquiescencia de tanta gente que no lo conseguirá nunca. Hay una cierta parálisis. (Entrevista 6. Responsable político del Gobierno local)

Una operación de reordenación de un sector que puede generar economías de escala y mayor eficacia en la gestión pública, pero que es interpretada como una agresión a la diversidad cultural y a la libertad creativa de los directores de los equipamientos culturales. Desde su punto de vista, el proceso de racionalización, que reconocen legítimo, no puede afectar a la identidad ni a la autonomía de cada institución cultural.

Las propuestas o alternativas se fundamentan en una lógica de racionalización o de mejora del sistema, con el deseo de fomentar la calidad. Pero cada centro cultural tiene una identidad, una función y unos objetivos diferenciados, hace una contribución específica a la cultura de la ciudad. [...] La diversidad es un componente esencial del ecosistema cultural y debe ser un requisito de las políticas culturales preservarla. Los cambios estructurales no pueden llegar por la vía de desvirtuar la identidad de los proyectos. Hay que mantener los modelos de éxito y dejar que los centros respondan a la crisis desde un modelo propio, sin imposiciones de modelos ajenos. La gestión de los proyectos hay

4. La polémica estalló cuando el director de la institución cultural manifestó, en una entrevista realizada en una cadena de radio nacional, querer cambiar el nombre del museo. Su propuesta se centraba en sustituir «Nacional de Catalunya» por «Nacional de Barcelona», para ganar proyección internacional. Tras un agrio debate y duras acusaciones por parte de sectores nacionalistas próximos al partido del Gobierno catalán (Convergència i Unió), el consejero de Cultura tuvo que contradecir públicamente al director el día después, afirmando que no se cambiaría el nombre, a pesar de que se había barajado previamente dicha posibilidad ( $E l$ País, 12 de agosto de 2012, 14). 
que enfocarlos desde criterios de expertos. (Entrevista 12. Director de institución cultural nacional)

En este caso, se opone la lógica racionalizadora que busca la eficiencia, la normalización y la ordenación de un sistema público orientado hacia el ciudadano con la lógica del mánager, que plantea la singularidad de su proyecto, defiende su éxito en términos culturales y exige la preeminencia de los criterios técnicos de los gestores culturales, todo ello por encima de los criterios políticos o de gestión pública.

Por otra parte, otro de los obstáculos a estos procesos de racionalización e intervención por parte de las administraciones públicas es el esquema de gobernanza compartido entre diversas administraciones públicas, un esquema muy común en el caso de Barcelona.

En el caso catalán y en el caso de Barcelona, como siempre ha habido este debate entre Barcelona y Cataluña, se ha construido un sistema con el que hasta ahora estaba bastante satisfecho, en el que se han creado consorcios entre las administraciones, compartiendo, de este modo, las responsabilidades. Ello ha generado mucha independencia y autonomía para estas instituciones culturales. El hecho que haya tensión entre las administraciones y que haya la presencia de dos, tres y hasta cuatro administraciones en el consejo de administración hace que todo quede bloqueado. Si bien ello ha garantizado una gestión autónoma, cosa que es básica, porque los directivos deben tener autonomía, hace también que no haya estrategias nuevas, porque todo hay que pactarlo entre todas las administraciones, y el consenso conlleva mediocridad. No hay apuesta, no hay riesgo, no hay fuerza. (Entrevista 5. Responsable político del Gobierno local)

Este esquema que se ha desarrollado durante veinte años, había sido percibido como un caso de éxito que había posibilitado la construcción y la puesta en funcionamiento de unas instituciones culturales a partir de una gobernanza multinivel compleja. No obstante, se trata de un sistema que, desde la perspectiva de los responsables políticos, desdibuja la tutela y la responsabilidad y convierte las instituciones culturales, en palabras del consejero de Cultura, en un «Franquenstein simpático pero ingobernable» (Mascarell, 2012). Así:

En cada institución artística, una administración pública debe ser preeminente y alguien debe tener la responsabilidad y ser el responsable final. Y sobre ello estamos haciendo un debate con el Ayuntamiento de Barcelona. El Museo Nacional de Arte de Cataluña debe tener preeminencia en la Generalitat. Y, en cambio, el Auditorio de Barcelona es perfectamente posible que sea responsabilidad del Ayuntamiento. [...] Ello daría mucha más claridad a la gestión. Es decir, el proceso para contratar el nuevo gerente del Auditorio ha sido un ejemplo de mala gestión. Se presentaron en el concurso veintinueve personas, ¡y fue declarado desierto! ¿Y todo ello por qué pasó?, porque las administraciones públicas no se pusieron de acuerdo. Hay muchas cosas a mejorar de esta situación. (Entrevista 3. Responsable político del Gobierno autonómico) 


\subsection{Dirigismo versus captura del interés público. Batalla simbólica}

A pesar del consenso sobre la necesidad de que se ejerza un mayor control de la gestión, los gestores también se alertan del riesgo de dirigismo en la política cultural, es decir, de una preponderancia del campo político sobre el campo artístico o de gestión interna. Ésta ha sido una acusación habitual (Moulin, 1992) que los mánagers culturales han utilizado como arma simbólica para defenderse de los intentos de mayor control de los responsables políticos.

El programa de gobierno puede entrar a planificar proyectos importantes, lo que no tiene sentido es que se acabe hablando de qué obras de teatro se tienen que programar. Esto es injerencia. Si lo que estamos tratando es que queremos hacer crecer los teatros, o trabajar para que se coordinen diversos teatros, o establecer nuevas líneas de trabajo educativo, todo ello se puede establecer en el contrato programa. Pero entrar más en detalle es injerencia a la tarea del director. (Entrevista 6. Responsable político del Gobierno local)

Pero, desde los responsables políticos, se alerta también de que un desequilibrio de poder hacia los mánagers podría derivar en lo que se llama «captura del interés público», es decir, una gestión orientada al beneficio de los que dirigen la organización, y no hacia la ciudadanía:

Un riesgo es que, como consecuencia de un encargo poco sólido por parte de las administraciones públicas, se produzca una apropiación gremial de la institución cultural. Todo el mundo critica a las apropiaciones políticas, pero nadie discute las apropiaciones por parte del sector artístico. Esta quiere decir que, en términos prácticos, el equipamiento deja de realizar la función para la que fue creado, la función colectiva o nacional. Entonces, aquí debemos encontrar el equilibrio. La Administración es la responsable del carácter público y debe contratar a directores en función del contrato programa. Debe dársele una autonomía de gestión, sí, pero luego debe auditarse de forma independiente y pública. (Entrevista 2. Responsable político del Gobierno autonómico)

Una de las tendencias en la relación entre responsables políticos y directores de las instituciones culturales es pasar de unas relaciones que se consideran demasiado normativizadas o burocratizadas y, por lo tanto, ineficaces o basadas en unas relaciones personales y, por lo tanto, clientelares, a unas relaciones basadas en la transparencia y la evaluación.

A nadie le parece extraño que se hagan auditorías económicas, pero, en cambio, nadie hace auditorías culturales. Y la auditoria tendría que fijarse no en el desajuste entre ingresos y gastos, sino entre proyecto y desarrollo de éste. Ahora hacemos una cosa que, a mi entender, es increíble: hacemos un concurso para elegir director, le contratamos y le decimos: «Invéntate el proyecto». No puede ser. El director no puede inventarse el proyecto, debe estar incardinado en el proyecto del director anterior y en el contrato programa. $Y$ al director hay que decirle: «Mire, usted debe moverse en estos parámetros». Y, además, debe marcarse unos objetivos de públicos. Otra cosa es que hará las exposiciones que 
quiera, pero esto no se audita nunca. Y una de las misiones del Consejo de la Cultura debe ser auditarlo. (Entrevista 1. Responsable político del Gobierno autonómico)

No obstante, como algunos autores han destacado, la evaluación en el sector de las organizaciones culturales es un asunto complejo por la vaguedad y la multiplicidad de los objetivos (Schuster, 1996). La solución adoptada tradicionalmente ha sido la de crear comisiones de expertos del mismo sector artístico que actuaban como una «academia invisible» para legitimar las elecciones de los responsables políticos (Urfalino, 1989). Por el contrario, actualmente se plantea que los proyectos artísticos sean valorados por el sector artístico, de modo que el Consejo de las Artes represente a posteriori los objetivos ya marcados anteriormente por parte de los responsables políticos, pero también esta posibilidad es contestada por los mánagers, que consideran que su tarea es mucho más compleja que una evaluación basada en indicadores y que su contribución al valor público debe basarse, en primer lugar, en una planificación interna.

El contrato programa será un primer paso. [...]. Pero esto no exime de tener un programa más ambicioso que es el plan estratégico, que es más ambicioso. Y toca algunas variables que son más intangibles. Y es que la Administración tiene un problema, que su lenguaje y sus mecanismos son anticuados. Ponen unas variables de evaluación que son obsoletas. El consejero dice: «Queremos un millón de visitantes», pero discutamos cómo se calculan: ¿cuentan o no los visitantes de la página web que están una hora leyendo? La exposición que va a Lleida, ¿cuenta o no cuenta? Los proyectos educativos que mandamos a las escuelas, ¿cuentan? ¿Y cuentan lo mismo los estudiantes de posgrado que hacen las clases aquí en el museo? Para la gran complejidad de la actividad, los mecanismos son muy rudimentarios. (Entrevista 12. Director de institución cultural nacional)

En definitiva, entre responsables políticos y mánagers de las instituciones culturales nacionales, existe una nueva articulación basada en la instrumentalización, la agencialización y la rendición de cuentas, así como nuevos conflictos para la definición sobre qué es y cómo se evalúa el valor público. Éste, y no la oposición entre arte y economía, es el objeto del debate simbólico — pero de efectos bien reales - sobre la legitimidad en la definición de los objetivos de gobernanza y la gestión de las instituciones culturales nacionales.

\section{Conclusiones}

Desde su construcción como instituciones de la alta cultura por parte de las élites sociales y económicas, las instituciones culturales nacionales han cambiado de función social y política (DiMaggio, 1991). La creciente interacción entre cultura y economía, las transformaciones de la estructura social y los cambios en los patrones de consumo cultural explican en buena parte estos cambios (Rodríguez Morató, 2007). Hasta mediados del siglo XX, en estas instituciones, 
se desarrollaba una oposición entre director artístico y gerente, figuras que representaban la articulación problemática entre un mundo artístico autónomo y la esfera de la economía (Moulin, 1983).

Actualmente, aunque esta oposición no ha desaparecido del todo, no constituye la problemática principal de la gestión de las instituciones culturales, porque la figura del mánager ha absorbido en gran parte los valores y los principios de actuación del mundo artístico (Boltanski y Chiapello, 2002; Chiapello, 1998), lo cual ha generado una concepción holística y proactiva de la gestión cultural (Mulcahy, 2003). Esta oposición ha sido substituida por los debates en torno a la articulación problemática entre los responsables políticos y los mánagers.

La crisis de legitimidad de las políticas culturales y su orientación instrumental posterior en pro de una nueva centralidad social (Gray, 2007) han provocado que, desde el campo político, se haya replanteado la misión de las grandes instituciones culturales, que hasta entonces se planteaba como definida desde el campo artístico y que, desde la Administración pública, estas instituciones se hayan conceptualizado como un instrumento de la política cultural, y no como entes completamente autónomos cuyas relaciones eran solamente de financiación (Coppinger et al., 2007).

En este contexto, asistimos a un giro en la gestión de las instituciones culturales, cuyos directores se convierten en mánagers que integran la visión artística y económica en una nueva predisposición proactiva e innovadora, orientada a la creación de valor público (Moore, 1995; Moore y Moore, 2005). Se trata de la nueva gestión pública, que defiende una mayor autonomía jurídica y financiera a cambio de una orientación a la gestión por objetivos y de una transparencia en la rendición de cuentas. Es por ello que asistimos a la proliferación de los instrumentos de fijación de los objetivos mediante contrato, como los contratos programa, que permiten combinar la autonomía de gestión con la definición de objetivos en mayor o menor medida entroncados con los de la política cultural definida desde la esfera política (Rius y Rubio, 2013).

Así pues, podemos decir que, en las instituciones culturales del siglo XXI, se desarrolla una nueva articulación entre responsables políticos y mánagers que sustituye a la antigua oposición entre director artístico y gerente. Como hemos podido ver, esta nueva articulación es un fenómeno reciente, presenta numerosos aspectos contradictorios y es percibida de forma en parte contradictoria en función de si se atiende al discurso generado por los mánagers o bien por los responsables políticos. Sin embargo, hemos mostrado que el debate sobre qué objetivos y formas debe caracterizar a esta articulación es un debate que, de forma progresiva, ocupa una mayor centralidad. En el caso de Barcelona, hemos podido constatar este fenómeno. Detrás de algunos puntos de consenso alrededor de la necesaria autonomía de gestión y de la libertad de los mánagers para desarrollar un proyecto, existen numerosos puntos de conflicto. Los procesos de reforma de la gestión de las redes de instituciones culturales, los cambios en el sistema de gobernanza, la negociación de los contratos programa o de sistema de evaluación de los resultados que los responsables políticos quieren 
introducir a cambio del soporte público generan reacciones significativamente diferenciadas en función de si el entrevistado es un responsable político o un mánager.

Hemos podido observar que existe un vivo debate y una oposición de visiones sobre dónde acaba la capacidad de tutela de los responsables de la Administración y dónde empieza la autonomía de gestión de los gerentes culturales. A la vez, también hay visiones contrapuestas sobre quién define la misión y los objetivos. Finalmente, si bien existe un consenso en que el objetivo de las instituciones culturales nacionales debe ser potenciar el valor público, también encontramos una batalla simbólica sobre cómo se define este valor público y quién lo evalúa. En este nuevo debate, los responsables políticos y los mánagers desarrollan discursos y estrategias para conseguir un predominio de visión sobre el valor público, que se ha convertido, así, en el nuevo "campo de batalla» de la política cultural y que refleja, a la vez, un nuevo reequilibrio entre la esfera artística y la esfera política (Dubois et al., 2012), en la que el rol del mánager gana centralidad, pero debe disputar con el representante político la legitimidad en la definición de la misión y el valor público de las instituciones culturales.

\section{Referencias bibliográficas}

Agid, Philippe y Tarondeau, Jean Claude (2006). L'Opéra de Paris: Gouverner une grande institution culturelle. París: Éditions Vuibert.

- (2007). «Governance of Major Cultural Institutions: The Case of the Paris Opera». International Journal of Arts Management, 10 (3), 4-18.

Ajuntament de Barcelona (2011). Anuari Estadístic de la Ciutat de Barcelona. Barcelona: Ajuntament de Barcelona.

ARTS COUnCIL ENGLAND (2011). Arts Council England's guidelines for boards of producing theatres appointing artistic leaders. Londres: Arts Council England.

Asuaga, Carolina y RAUSELl, Pau (2006). «Un Análisis de la gestión de instituciones culturales: El caso específico de los museos». Revista Iberoamericana de Contabilidad de Gestión, 8 (4), 83-104.

BECKER, H. S. (1984). Art Worlds. Berkeley: University of California Press.

BELFIORE, Eleonora (2004). «Auditing Culture: The subsidised cultural sector in the New Public Management». International Journal of Cultural Policy, 10 (2), 183-202. <http://dx.doi.org/10.1080/10286630042000255808>

- (2006). «The social impact of the arts - myth or reality». En: MiRZA, M. (ed.). Culture Vultures: Is UK arts policy damaging the arts? Londres: Policy Exchange.

BiANCHINI, Franco (1993). «Remaking European Cities: The role of cultural policies». En: Bianchini, F. y PARKInson, M. (eds.). Cultural Policy and Urban Regeneration: The West European Experience. Manchester: Manchester University Press, 1-19.

BILTON, Chris (2006). Management and Creativity: From the creative industries to creative management. Oxford: Blackwells.

BILTON, Chris y LEARY, Ruth (2002). «What can managers do for creativity?: Brokering creativity in the creative industries». International Journal of Cultural Policy, 8 (1), 49-64. $<$ http://dx.doi.org/10.1080/10286630290032431> 
BoltANSKI, Luc y ChIAPELlO, Eve (2002). El nuevo espiritu del capitalismo. Madrid: Akal. Chiapello, Eve (1998). Artistes versus managers: Le management culturel face à la critique artiste. París: Métailié: Diffusion, Seuil.

Coppinger, Nathalie; Joder, Mélanie; LeCA, Charlotte y Dupuit, Jean-Sébastien (2007). Rapport sur la tutelle et le pilotage des opérateurs au ministère de la culture. París: Ministère de l'économie, des finances et de l'industrie.

Cray, David; Inglis, Loretta y FreEMAN, Susan (2007). «Managing the Arts: Leadership and Decision Making under Dual Rationalities». Journal of Arts Management, Law and Society, 36 (4), 295-313.

<http://dx.doi.org/10.3200/JAML.36.4.295-314>

Crozier, Michel y Friedberg, Erhard (1982). L'Acteur et le système: Les contraintes de l'action collective. París: Éditions du Seuil.

Departament de Cultura (2011). Memoria del Departament de Cultura 2010. Barcelona: Departament de Cultura. Generalitat de Catalunya.

DEWEY, Patricia (2004). «From arts management to cultural administration». International Journal of Arts Management, 6 (3), 13-22.

Dimaggio, Paul (1987a). Managers of the Arts. Washington DC: National Endowment for the Arts. Seven Locks Press.

- (1987b). «Nonprofit Organizations in the Production and Distribution of Culture». En: Powell, W. W. (ed.). The Nonprofit Sector: A Research Handbook. New Haven: Yale University Press, 195-220.

- (1991). «Social Structure, Institutions and Cultural Goods: The Case of the United States». En: Bourdieu, P. y Coleman, J. (eds.). Social Theory for a Changing Society. Boulder: Westview Press, 133-166.

Dubois, Vincent; Bastien, Clément; Fryermuth, Audrey y Matz, Kévin (2012). La politique, l'artiste et le gestionnaire: (Re)configurations locales et (dé)polisation de la culture. Bellecombe-en-Bauges: Éditions du Coquant.

Evans, G. (2003). "Hard-Branding the cultural city-from Prado to Prada». International Journal of Urban and Regional Research, 27 (2), 417-440. <http://dx.doi.org/10.1111/1468-2427.00455>

Evrard, Yves y ColberT, François (2000). «Arts Management: A New Discipline Entering the Millennium?». International Journal of Arts Management, 2 (2), 4-13.

Gray, Clive (2007). "Commodification and instrumentality in cultural policy». International Journal of Cultural Policy, 13 (2), 203-215. <http://dx.doi.org/10.1080/10286630701342899>

- (2008). «Instrumental policies: Causes, consequences, museums and galleries». Cultural Trends, 17 (4), 209-222. <http://dx.doi.org/10.1080/09548960802615349>

Holden, John (2004). Capturing cultural value. Londres: Demos.

- (2008). Democratic Culture. Opening Up the Arts for Everyone. Londres: Demos.

Kuesters, Ivonne (2010). "Arts Managers as Liaisons between Finance and Art: A Qualitative Study Inspired by the Theory of Functional Differentiation». The Journal of Arts Management, Law and Society, 40, 43-57. <http://dx.doi.org/10.1080/10632921003603976>

LASH, Scott (1990). Sociology of Postmodernism. Londres: Routledge.

LEE, David John; OAKLeY, Kate y NAYLOR, Richard (2011). "The public gets what the public wants"? The uses and abuses of "public value" in contemporary British cultural policy». International Journal of Cultural Policy, 17 (3), 289. <http://dx.doi.org/10.1080/10286632.2010.528834> 
LONGO, Francisco (2007). «Introducció: Els directius públics davant els reptes de la governança contemporània». En: LONGO, F. e YsA, T. (eds.). Els escenaris de la gestió pública del segle XXI. Escola d'Administració Pública de Catalunya, 15-32.

MARSHALL, Tim (1996). "Barcelona fast forward?: City entrepreneurialism in the 1980s and 1990s». European Planning Studies, 4 (2), 147-165. <http://dx.doi.org/10.1080/09654319608720337>

Martorella, Roseanne (1982). The Sociology of Opera. Nueva York: Bergin Publishers.

Mascarell, Ferran (2012). "Compareixença de l'Honorable Conseller de Cultura Ferran Mascarell». Comissió de Cultura del Parlament de Catalunya, 11, 9-37.

MENDOZA, Xavier y VERnIS, Alfred (2007). "L'Estat relacional i la transformació de les administracions públiques». En: LongO, F. e YSA, T. (eds.). Els escenaris de la gestió pública del segle XXI. Barcelona: Escola d'Administració Pública de Catalunya, 33-55.

Menger, Piere-Michel (2009). Le travail créateur: S’accomplir dans l'incertain. París: Gallimard.

Moore, Mark H. (1995). Public Value: Strategic Management in Government. Boston: Harvard University Press.

Moore, Mark H. y Moore, Gaylen W. (2005). Creating Public Value Through State Arts Agencies. Minneapolis: Arts Midwest.

Moulin, Raymond (1983). Le marché de l'art en France. París: Éditions de Minuit.

- (1992). L'artiste, l'institution et le marché. París: Flammarion.

Mulcahy, Kevin (1998). "Cultural Patronage in Comparative Perspective: Public Support for the Arts in France, Germany, Norway, and Canada». Journal of Arts Management, Law and Society, 27 (4), 247-263.

<http://dx.doi.org/10.1080/10632929809597270>

- (2003). "Entrepreneurship or Cultural Darwinism?: Privatisation and American Cultural Patronage». Journal of Arts Management, Law and Society, 33 (3), 165-184.

Peterson, Richard A. (1986). "From Impresario to Art Administrator: Formal Accountability in Nonprofit Cultural Organizations». En: DiMaggio, P. (ed.). Nonprofit enterprises in the arts: Studies in mission and constraint. Nueva York: Oxford University Press, 161-183.

RiUs Uldemollins, Joaquim (2008). «Los barrios artísticos como base local de la cultura global: El caso del Raval de Barcelona». Revista Internacional de Sociología, 66 (51), 179-205.

RiUS, Joaquim (2005). Un nou paradigma de la política cultural: Estudi sociologic del cas Barcelona. Barcelona: Universitat Autònoma de Barcelona / École des Hautes Études en Sciences Sociales.

- (2006). «El MACBA i el CCCB: De la regeneració cultural a la governança cultural». Digithum, 8, 10-17.

- (2010). Els contractes programa dels equipaments culturals: Governança, eficiència $i$ retorn social del sistema públic d'equipaments culturals de Catalunya. Barcelona: Departament de Cultura i Mitjans de Comunicació.

- (2012a). "Gallery Districts of Barcelona: The Strategic Play of Art Dealers». The Journal of Arts Management, Law and Society, 42 (2), 48-62. <http://dx.doi.org/10.1080/10632921.2012.678929>

- (2012b). «Política cultural e hibridación de las instituciones culturales: El caso de Barcelona». Revista Española de Ciencia Política, 29, 85-105.

RiUS, Joaquim y RUBIO, Arturo (2013). «The governance of national cultural organisations: comparative study of performance contracts with the main cultural organisa- 
tions in England, France and Catalonia». International Journal of Cultural Policy, 19 (2), 249-269.

<http://dx.doi.org/10.1080/10286632.2011.638981>

ROdRíGUEZ Morató, Arturo (2005). «La reinvención de la política cultural a escala local: El caso de Barcelona». Sociedade e Estado, 20 (2), 351-376. $<$ http://dx.doi.org/10.1590/S0102-69922005000200005>

- (2007). «La perspectiva de la sociedad de la cultura». En: La sociedad de la cultura. Barcelona: Ariel, 11-51.

- (2008). «La emergencia de una capital cultural europea». En: DEGEN, M. y GARCíA, S. (eds.). La metaciudad: Barcelona, transformación de una metrópolis. Barcelona: Anthropos.

SCHUSTER, J. Mark (1996). «The performance of performance indicators in the arts». Nonprofit Management and Leadership, 7 (3), 253-269. $<$ http://dx.doi.org/10.1002/nml.4130070304>

- (1998). «Neither Public Nor Private: The Hybridization of Museums». Journal of Cultural Economics, 22 (2-3), 127-150.

StOne, Melissa M. y SANDFORT, Jodi R. (2009). «Building a Policy Fields Framework to Inform Research on Nonprofit Organizations». Nonprofit and Voluntary Sector Quarterly, 38 (6), 1054-1075. <http://dx.doi.org/10.1177/0899764008327198>

Trullén, J. (2001). «El projecte Barcelona: Ciutat del coneixement des de l'economia». Barcelona Metrópolis Mediterrániarcelona, 1, 16-25.

URFAlino, Philippe (1989). «Les politiques culturelles: Mécénat caché et académies invisibles». L'Anne Sociologique, 3 (39), 81-109.

Villarroya, Anna (2012). "Cultural policies and national identity in Catalonia». International Journal of Cultural Policy, 18 (1), 31-45. $<$ http://dx.doi.org/10.1080/10286632.2011.567330>

Weber, Max (1944). Economía y sociedad. México: Fondo de Cultura Económica.

- (1998). Ensayos sobre sociología de la religión. Madrid: Taurus.

White, Harrison C. y White, Cynthia A. (1991). La Carrière des peintres au XIXe siècle: Du système académique au marché des impressionnistes. París: Flammarion.

Zimmer, Annette y Toepler, Stefan (1999). «The Subsidized Muse: Government and the Arts in Western Europe and the United States». Journal of Cultural Economics, 23, 33-49. <http://dx.doi.org/10.1023/A:1007565515785>

ZOLBERG, Vera L. (2007). «Los retos actuales de la política cultural: una nueva idea de comunidad». En: Rodríguez Morató, A. (ed.). La sociedad de la cultura. Barcelona: Ariel, 89-105. 